\title{
Overexpression of mitochondrial serine hydroxyl- methyltransferase 2 is associated with poor prognosis and promotes cell proliferation and invasion in gliomas
}

\author{
This article was published in the following Dove Press journal: \\ OncoTargets and Therapy \\ 27 July 2017 \\ Number of times this article has been viewed
}

Ming Wu

Siyi Wanggou

Xuejun Li

Qing Liu

Yuanyang Xie

Department of Neurosurgery, Xiangya Hospital, Central South University, Changsha, Hunan,

People's Republic of China
Correspondence: Yuanyang Xie Department of Neurosurgery, Xiangya Hospital, Central South University, 87 Xiangya Road, Kaifu District, Changsha, Hunan 410008, People's Republic of China Tel/fax+8673I8265000 Email deanxyy@I26.com

\begin{abstract}
Mitochondrial serine hydroxyl-methyltransferase 2 (SHMT2), participating in the synthesis of mitochondrial thymidine monophosphate, has been reported to drive glioma cell survival in ischemia. However, its clinical relevance in gliomas remains unclear. In the current study, immunohistochemistry was performed to examine subcellular localization and expression levels of SHMT2 protein in glioma and non-neoplastic brain tissue specimens. Then, the associations of SHMT2 expression with various clinicopathological features and patients' prognosis were statistically evaluated. The roles of SHMT2 in the proliferation and invasion of glioma cells after siRNA-SHMT2 vector transfection were also detected by cell counting kit- 8 and transwell assays, respectively. Results showed that SHMT2 immunostaining was predominantly localized in the cellular cytoplasm of tumor cells in glioma tissues but weakly in non-neoplastic brain tissues. Statistically, SHMT2 protein expression was significantly higher in glioma tissues than in non-neoplastic brain tissues $(P<0.001)$. In addition, SHMT2 overexpression more frequently occurred in glioma patients with an advanced grade of malignancy $(P<0.001)$ and poor prognosis $(P=0.001)$. Notably, multivariate analysis based on a Cox regression model identified SHMT2 expression as an independent prognostic factor for glioma patients $(P=0.01)$. Functionally, SHMT2 knockdown efficiently suppressed the proliferation $(P=0.02)$ and invasion $(P<0.001)$ of glioma cells in vitro. In conclusion, our findings suggest that SHMT2 may function as an oncogene in glioma development and progression. Clinically, SHMT2 may serve as a prognostic factor and as a potential therapeutic target for human gliomas.
\end{abstract}

Keywords: mitochondrial serine hydroxyl-methyltransferase 2, glioma, prognosis, oncogene, cell proliferation, cell invasion

\section{Introduction}

Human gliomas, a group of the most lethal tumors in the central nervous system (CNS), account for $\sim 70 \%$ of malignant brain tumors. ${ }^{1}$ On the basis of cytologic features and degree of malignancy, gliomas are categorized into grades I, II, III, and IV according to the World Health Organization (WHO) classification: grade I, pilocytic astrocytoma, is indicated to be biologically benign tumor; grade II, diffuse astrocytoma, is considered as a low-grade malignancy and is associated with prolonged survival; and both grade III (anaplastic astrocytoma) and grade IV (glioblastoma [GBM]) are highly malignant tumors, which can lead to unsatisfactory clinical outcomes. ${ }^{2,3}$ Although there have been great advances in brain tumor therapy, including surgical resection, chemotherapy and radiotherapy, the prognosis of glioma patients remains dismal. ${ }^{4}$ 
In particular, GBM patients have a median survival time of only 9-12 months. ${ }^{5}$ Moreover, growing clinical evidence shows that similar clinicopathological features of glioma patients may exhibit different responses to therapy and various prognoses. Therefore, it is necessary to clarify the underlying molecular mechanisms and to identify novel tumor biomarkers in order to enhance early diagnosis, determine novel therapeutic targets and improve the clinical outcomes of glioma patients.

Mitochondrial serine hydroxyl-methyltransferase 2 (SHMT2), localized in 12q13, is a mitochondrial enzyme that is predominantly expressed in the mitochondrion, but it has also been observed in the cytoplasm and nucleus. ${ }^{6}$ SHMT2, responsible for catalyzing the conversion of serine to glycine with the transfer of $\beta$-carbon from serine to tetrahydrofolate (THF) to form 5,10-methylene-THF, acts as a crucial factor for the serine/glycine metabolism in proliferating cells. ${ }^{7}$ Accumulating studies have reported that abnormal expression of SHMT2 may play an important role in tumorigenesis and tumor progression of various human malignancies. ${ }^{8-11}$ Especially, Kim et al ${ }^{12}$ indicated that SHMT2 was elevated in a subset of glioma cells and promoted changes in metabolism that allowed cells to survive in an ischemic tumor microenvironment. However, its clinical relevance in human gliomas remains unclear.

To address this problem, subcellular localization and expression level of SHMT2 protein were examined by immunohistochemistry using glioma and non-neoplastic brain tissue specimens. Then, the associations of SHMT2 expression with various clinicopathological features and patients' prognosis were statistically evaluated. Moreover, the effects of SHMT2 on the proliferation and invasion of glioma cells transfected with siRNA-SHMT2 vector were detected by cell counting kit-8 (CCK-8) and transwell assays in vitro, respectively.

\section{Patients and methods}

\section{Patients and tissue samples}

The present study was approved by the research ethics committee of Xiangya Hospital according to the Declaration of Helsinki. All patients provided written informed consent, and all tissue specimens were handled and made anonymous in accordance with the ethical and legal standards.

To detect the subcellular localization and expression levels of SHMT2 protein in glioma tissues by immunohistochemistry analysis, 162 formalin-fixed, paraffin-embedded specimens of gliomas collected between 2001 and 2011 were retrieved from the archives of Department of Pathology,
Xiangya Hospital. On the basis of the WHO classification assessed by two pathologists, there were 20, 26, 50 and 66 patients with WHO grade I, II, III and IV, respectively. As a control group, 20 paraffin and snap-frozen sections of non-neoplastic brain tissues from 20 patients with intractable epilepsy were also collected. All the patients enrolled in this study did not have a history of other tumors and did not receive chemotherapy or radiotherapy before the surgical operation. The clinicopathological features and the treatment strategies of all the patients are summarized in Table 1.

All 162 glioma patients had complete follow-up until death, with a median follow-up period of 21.6 months (range 0.5-70 months). During the follow-up, glioma patients were monitored every 2-3 months by clinical interview or phone call. Patients who died of diseases not directly related to gliomas or due to unexpected events were excluded at the case collection stage in this study. Overall survival was defined as the duration from the date of the initial surgical operation to death.

\section{Immunohistochemistry analysis}

Immunohistochemistry analysis was performed to examine the subcellular localization and expression pattern of SHMT2 protein in human glioma and non-neoplastic brain tissues according to the previous studies. ${ }^{13,14}$ Following antigen retrieval, the sections were added with $0.3 \%$ hydrogen peroxide for $30 \mathrm{~min}$ to suppress endogenous peroxidase activities and blocked with $2 \%$ goat serum for $30 \mathrm{~min}$. After that, the sections were blocked with $2 \%$ goat serum

Table I Clinicopathological characteristics of I 62 glioma patients

\begin{tabular}{|c|c|c|c|c|}
\hline \multirow[t]{2}{*}{ Features } & \multicolumn{4}{|c|}{ WHO classification grading } \\
\hline & WHO & | WHO II & WHO III & WHO IV \\
\hline No of cases & 20 & 26 & 50 & 66 \\
\hline Mean age (years) & 39.8 & 46.8 & 46.2 & 49.8 \\
\hline \multicolumn{5}{|l|}{ Gender, $\mathrm{n}$} \\
\hline Male & 14 & 16 & 35 & 46 \\
\hline Female & 6 & 10 & 15 & 20 \\
\hline \multicolumn{5}{|l|}{ KPS score, $n$} \\
\hline$\geq 80$ & 15 & 20 & 11 & 16 \\
\hline$<80$ & 5 & 6 & 39 & 50 \\
\hline \multicolumn{5}{|l|}{ Surgery, n } \\
\hline Gross total resection & 20 & 26 & 38 & 46 \\
\hline Partial resection & 0 & 0 & 9 & 15 \\
\hline Biopsy & 0 & 0 & 3 & 5 \\
\hline \multicolumn{5}{|l|}{ Adjuvant treatment, $\mathrm{n}$} \\
\hline Radiotherapy & 0 & 0 & 32 & 15 \\
\hline Chemotherapy & 0 & 2 & 0 & 10 \\
\hline Radiotherapy and & 0 & 0 & 8 & 30 \\
\hline
\end{tabular}

Abbreviations: WHO, World Health Organization; KPS, Karnofsky performance score. 
for $30 \mathrm{~min}$ and incubated overnight at $4^{\circ} \mathrm{C}$ with polyclonal rabbit anti-human SHMT2 antibody (1:150, ab180786; Abcam, Cambridge, MA, USA). The specificity of SHMT2 antibody was confirmed using peptide blocking assay. For negative control, rabbit IgG was used in place of a primary rabbit polyclonal antibody. Following the incubation with the horseradish peroxidase-conjugated secondary antibody (1:500; Abcam), the sections were incubated using a 3,3'diaminobenzidine tetrahydrochloride (DAB) and counter stained with hematoxylin, dehydrated and cover slipped.

Two independent pathologists, who were both blinded to the patient background, were invited to evaluate SHMT2 immunostaining using immunoreactive score (IRS), which was calculated by multiplying the staining intensity and the positive cells' percentage scores according to the previous studies. ${ }^{13,14}$ Staining intensity was scored as follows: "0", negative; "1", weakly positive; "2", moderately positive and " 3 ", strongly positive. The percentage of SHMT2-positive cells was scored as $0(0 \%), 1(1 \%-25 \%), 2(26 \%-50 \%)$ and $3(>50 \%)$. Images were taken from three fields, and the mean IRS value was calculated for each case. To evaluate the associations of SHMT2 expression with various clinicopathological features and patients' prognosis, the median value of SHMT2 protein IRS in all glioma tissue sections was used as a cutoff point to divide 162 glioma patients into SHMT2-low and -high groups.

\section{Cell culture and transfection}

Human glioma cell lines U87 and H4 were purchased from the Chinese Academy of Sciences Cell Bank (Beijing, China). Two cell lines were cultured using Dulbecco's Modified Eagle's Medium (DMEM; Gibco BRL Co. Ltd., San Fransisco, CA, USA) supplemented with $10 \%$ fetal bovine serum (FBS; Gibco BRL Co. Ltd.) and were maintained at $37^{\circ} \mathrm{C}$ in a humidified chamber supplemented with $5 \% \mathrm{CO}_{2}$.

To determine the function of SHMT2 in malignant phenotypes of glioma cells, sequence-specific siRNA to SHMT2 (si-SHMT2; 5'-CGG AGA GTT GTG GAC TTT ATA-3') and control siRNA (si-con; 5'-ACA ACA GCC ACA ACG TCT ATA-3') were purchased from GenePharma (Shanghai, China). Cells $\left(5 \times 10^{5}\right)$ were transfected in six-well dishes for 3-4 h with the RNA duplexes (200 nM) using Lipofectamine 2000 (Life Technologies, Carlsbad, CA, USA) according to the instructions of the manufacturer.

\section{Western blot}

Western blot analysis was performed to detect the expression level of SHMT2 protein in glioma cells with the transfection of si-SHMT2 according to the previous studies. ${ }^{15,16}$ Glioma cell pellets were harvested after being transfected with SHMT2-specific siRNA for $48 \mathrm{~h}$ and were lysed with high potassium chloride $(\mathrm{KCl})$ lysis buffer with complete protease inhibitor cocktail (Roche, Nutley, NJ, USA). Protein concentration was qualified by Bicinchoninic Acid Protein Assay Kit (Pierce, Rockford, IL USA). Equal amount of proteins was separated on a $10 \%$ sodium dodecyl sulfate polyacrylamide gel electrophoresis (SDS-PAGE) and then transferred to polyvinylidene fluoride or polyvinylidene difluoride (PVDF) membranes, which subsequently were blocked with $3 \%$ bovine serum albumin (BSA) for $2 \mathrm{~h}$ at room temperature. Then, the blots were incubated with the primary antibodies against SHMT2 (1:250, ab180786; Abcam) or $\beta$-actin (as an internal loading control, 1:200, ab8227; Abcam) at $4^{\circ} \mathrm{C}$ overnight. After that, the bands were visualized with Chemiluminescence Detection Kit (GE Healthcare, Milwaukee, WI, USA) and analyzed by Quantity One software (Bio-Rad, Hercules, CA, USA). The relative amount of SHMT2 protein was determined by normalizing the densitometry value of interest to that of loading control $\beta$-actin.

\section{CCK-8 assay}

The CCK-8 assay was performed to determine the role of SHMT2 in cell proliferation of human glioma cells according to a previous study. ${ }^{17}$ In brief, glioma cells were incubated with $20 \mu \mathrm{L}$ of CCK-8 solution (Beyotime Biotechnology, Shanghai, China) for $4 \mathrm{~h}$ at $37^{\circ} \mathrm{C}$, after being cultured for 24 , 48 and $72 \mathrm{~h}$. The absorbance was measured at the wavelength of $495 \mathrm{~nm}$ with a spectrophotometer. The experiments were carried out in three independent experiments.

\section{Transwell assay}

Transwell assay was performed to determine the role of SHMT2 in cell invasion of human glioma cells using transwell filters with Matrigel according to the previous studies. ${ }^{17}$ The invaded cells were fixed in $100 \%$ methanol for $10 \mathrm{~min}$, air dried and then stained with 4',6-diamidino-2-phenylindole (DAPI) before being counted under a microscope. The number of invaded cells in five fields was counted under a magnification of $200 \times$, and the mean values for each chamber were determined.

\section{Statistical analysis}

All statistical analyses in the present study were performed using the SPSS software for Windows (version 13.0; SPSS Inc., Chicago, IL, USA). Continuous variables were expressed as mean \pm SD. Data obtained from immunohistochemistry 
and Western blot analysis were conducted using the Wilcoxon signed-rank test. The Kaplan-Meier method was used for the survival analysis, and Cox regression analysis was used for the univariate and multivariate analyses. Statistical analyses of in vitro cell proliferation and invasion assays were performed using the two-tailed Student's $t$-test. $P$-values $<0.05$ were considered as statistically significant.

\section{Results}

\section{SHMT2 protein overexpression in human glioma tissues}

Of 162 glioma patients, 56 (34.57\%), 72 (44.44\%), 22 $(13.58 \%)$ and 12 cases $(7.41 \%)$ displayed expression of SHMT2 as strongly positive, moderately positive, weakly positive and negative, respectively, in the cytoplasm of tumor cells (Figure 1A-D), while 20 non-neoplastic brain tissues showed weak or negative expression of SHMT2 protein (Figure 1E). Statistically, SHMT2 protein expression in glioma tissues was significantly higher than that in non-neoplastic brain tissues (tumor vs normal: $5.80 \pm 1.71$ vs 2.35 $\pm 0.63, P<0.001$; Figure $1 \mathrm{~F})$.

\section{SHMT2 protein overexpression associates with aggressive tumor progression of human gliomas}

All 162 glioma patients were divided into SHMT2-low $(n=80,49.38 \%)$ and SHMT2-high $(n=82,50.62 \%)$ groups using the median value (5.49) of SHMT2 IRS in glioma tissues as a cutoff point. As shown in Table 2, glioma patients with high SHMT2 expression more frequently had advanced WHO grade (III-IV) than those with low SHMT2 expression $(P<0.001)$. However, there were no differences in statistical significance between SHMT2 expression and other clinicopathological characteristics, including patients' age and gender and Karnofsky performance score (KPS) (all $P>0.05$; Table 2).

\section{SHMT2 protein overexpression predicts poor prognosis of patients with gliomas}

Kaplan-Meier survival curves illustrated a significant difference in overall survival between SHMT2-low and -high glioma patient groups $(P=0.001$, by the log-rank test; Figure 2). Further multivariate analysis was performed to
A

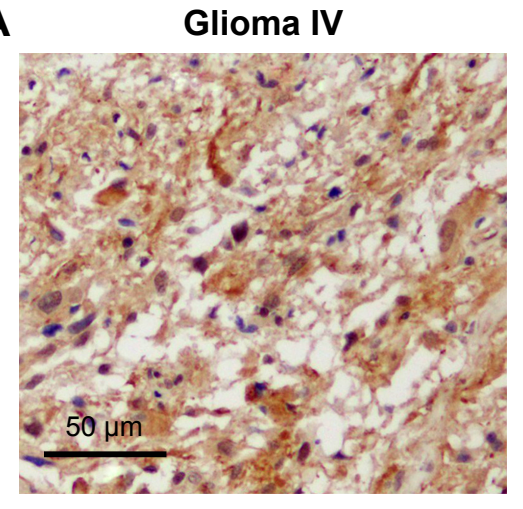

D

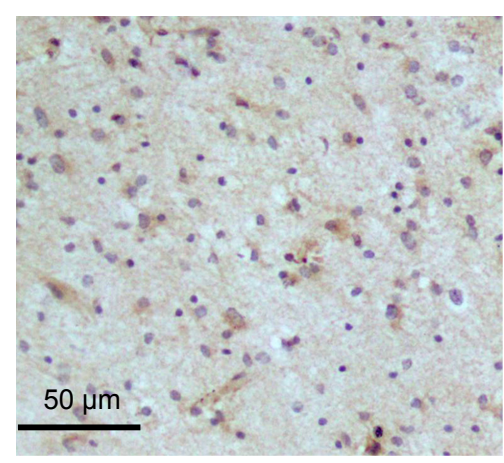

B

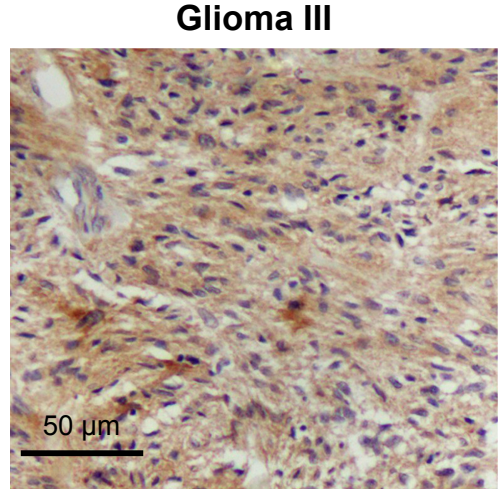

E

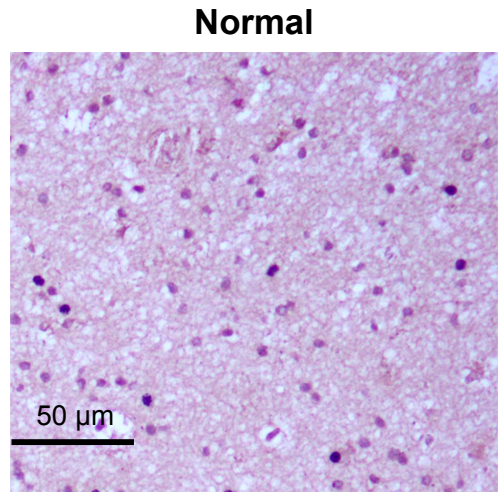

C

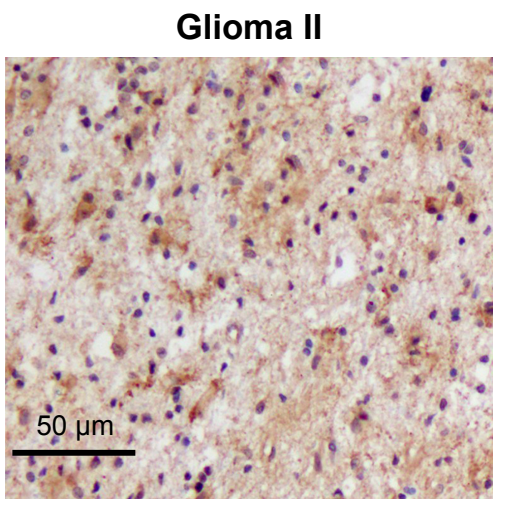

$\mathbf{F}$

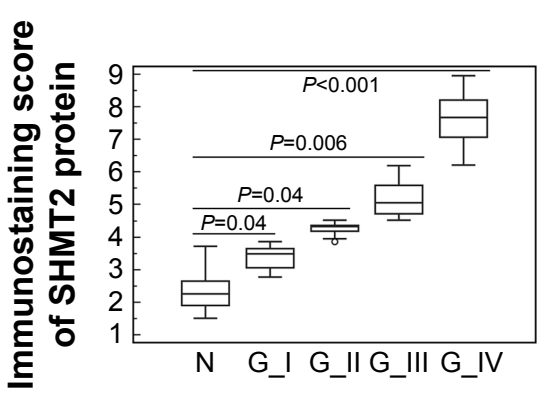

Figure I Expression pattern and subcellular localization of SHMT2 protein in human glioma and non-neoplastic brain tissues examined by immunohistochemistry. Notes: (A-E) Positive immunostainings of SHMT2 protein were mainly observed in the cytoplasm of tumor cells in grade I-IV glioma tissues, but negative or weak immunostaining was shown in non-neoplastic brain tissues (magnification 200x). (F) Statistical analysis of SHMT2 protein immunostaining in glioma and non-neoplastic brain tissues. "N" refers to non-neoplastic brain tissues, G_I refers to glioma tissues with WHO grade I, G_II refers to glioma tissues with WHO grade II, G_III refers to glioma tissues with WHO grade III and G_IV refers to glioma tissues with WHO grade IV.

Abbreviations: SHMT2, serine hydroxyl-methyltransferase 2; WHO, World Health Organization. 
Table 2 Associations of SHMT2 protein expression with various clinicopathological characteristics of glioma patients

\begin{tabular}{|c|c|c|c|c|}
\hline $\begin{array}{l}\text { Clinicopathological } \\
\text { characteristics }\end{array}$ & $\begin{array}{l}\text { No of } \\
\text { cases }\end{array}$ & $\begin{array}{l}\text { SHMT2-low, } \\
\text { n (\%) }\end{array}$ & $\begin{array}{l}\text { SHMT2-high, } \\
\text { n (\%) }\end{array}$ & $P$-value \\
\hline WHO grade & & & & $<0.001$ \\
\hline I & 20 & $20(100.0)$ & $0(0)$ & \\
\hline II & 26 & $18(69.23)$ & $8(30.77)$ & \\
\hline III & 50 & $26(47.27)$ & $24(52.73)$ & \\
\hline IV & 66 & $16(24.24)$ & $50(75.76)$ & \\
\hline Age (years) & & & & NS \\
\hline$<55$ & 82 & $45(54.88)$ & $37(45.12)$ & \\
\hline$\geq 55$ & 80 & $35(43.75)$ & $45(56.25)$ & \\
\hline Gender & & & & NS \\
\hline Male & 111 & $55(49.55)$ & $56(50.45)$ & \\
\hline Female & 51 & $25(49.02)$ & $26(50.98)$ & \\
\hline KPS score & & & & NS \\
\hline$<80$ & 100 & $42(42.00)$ & $58(58.00)$ & \\
\hline$\geq 80$ & 62 & $38(61.29)$ & 24 (38.7I) & \\
\hline
\end{tabular}

Note: "NS" refers to the differences without statistical significance.

Abbreviations: SHMT2, serine hydroxyl-methyltransferase 2; WHO, World Health Organization; KPS, Karnofsky performance score.

evaluate the prognostic value of SHMT2 and various clinicopathological characteristics by using the Cox regression model. Data in Table 3 indicated that the WHO grade (hazard ratio $4.92 ; 95 \%$ confidence interval $[\mathrm{CI}] 1.98-11.21 ; P=0.01)$ and SHMT2 protein expression (hazard ratio $4.36 ; 95 \% \mathrm{CI}$ $1.82-9.82 ; P=0.01$ ) were both independent prognostic factors for overall survival of glioma patients.

\section{SHMT2 regulates cell proliferation and invasion of glioma cells in vitro}

To further explore the effects of SHMT2 on cell proliferation and cell invasion, the endogenous expression of SHMT2 was

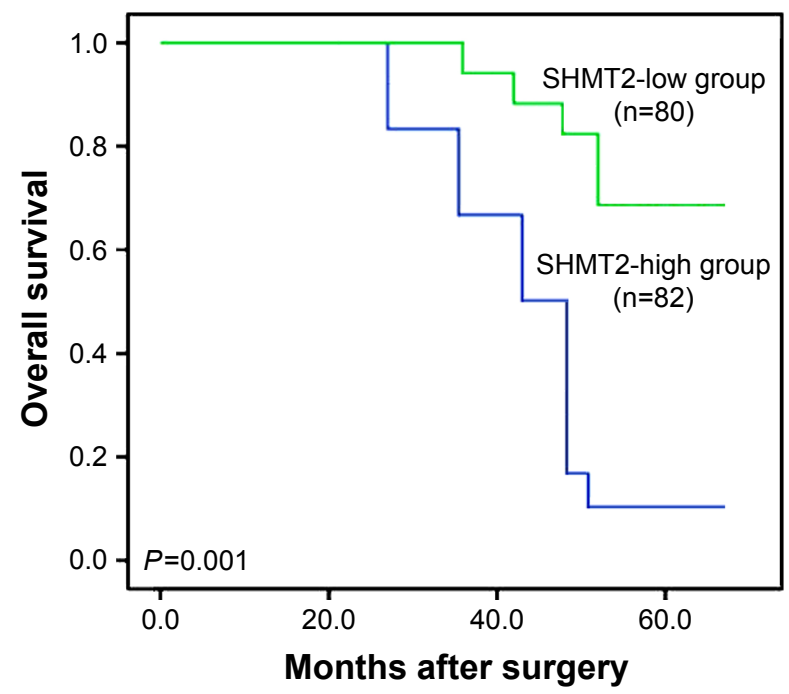

Figure 2 Kaplan-Meier survival curves of glioma patients based on the expression levels of SHMT2 protein.

Abbreviation: SHMT2, serine hydroxyl-methyltransferase 2.
Table 3 Cox multivariate analysis on the associations of various clinicopathological characteristics with patients' overall survival

\begin{tabular}{llll}
\hline Features & Hazard ratio & $\mathbf{9 5 \%} \mathbf{C l}$ & P-value \\
\hline Age & 1.08 & $0.66-1.92$ & $\mathrm{NS}$ \\
Gender & 0.92 & $0.58-1.68$ & $\mathrm{NS}$ \\
WHO grade & 4.92 & $1.98-11.21$ & $0.0 \mathrm{I}$ \\
KPS score & 1.26 & $1.08-2.69$ & $\mathrm{NS}$ \\
Extent of resection & 1.32 & $1.19-2.92$ & $\mathrm{NS}$ \\
Type of adjuvant treatment & 1.33 & $1.12-2.88$ & $\mathrm{NS}$ \\
SHMT2 expression & 4.36 & $1.82-9.82$ & 0.01 \\
\hline
\end{tabular}

Note: "NS" refers to the differences without statistical significance. Abbreviations: $\mathrm{Cl}$, confidence interval; WHO, World Health Organization; KPS, Karnofsky performance score; SHMT2, serine hydroxyl-methyltransferase 2.

knocked down in U87MG and H4 cells using the specific si-SHMT2 and the transfection efficiency was validated by Western blot analysis. As shown in Figure 3A, the siRNA against SHMT2 had the effective inhibition for the expression of the corresponding protein $(P<0.001)$.

Functionally, CCK-8 and transwell assays were performed to measure the response of two glioma cell lines to the siRNA specific to SHMT2. After being cultured for $72 \mathrm{~h}$, the cell proliferative activities of si-SHMT2-transfected U87MG and $\mathrm{H} 4$ cells were both markedly decreased compared to those of si-con-transfected cells (Figure 3B for U87MG cells and Figure 3C for $\mathrm{H} 4$ cells, both $P<0.05$ ). Moreover, transwell assays also indicated that the downregulation of SHMT2 efficiently reduced the cell invasion of both $\mathrm{U} 87 \mathrm{MG}$ and $\mathrm{H} 4$ cells compared to that of control cells (all $P<0.05$; Figure 4).

\section{Discussion}

Characterized with high rates of morbidity and mortality, gliomas have been a great challenge in the research field of CNS tumors, regardless of various efforts to develop new diagnosis and therapeutic strategies. ${ }^{18}$ Accumulating studies have indicated that the uncontrollable proliferation, migration and invasion of glioma cells may be the main reasons for unfavorable prognosis of patients with this tumor. ${ }^{19,20}$ To investigate the underlying molecular mechanisms that determine the biological behaviors of glioma cells may be very important to identify new drug targets and to develop efficient therapeutic methods for controlling the malignant phenotypes of gliomas. In the present study, our immunohistochemistry analysis observed that SHMT2 immunostaining was predominantly localized into cellular cytoplasm of tumor cells in glioma tissues, but weakly in non-neoplastic brain tissues. Statistically, SHMT2 protein expression in glioma tissues was significantly higher than that in nonneoplastic brain tissues $(P<0.001)$. In addition, SHMT2 overexpression more frequently occurred in glioma patients 

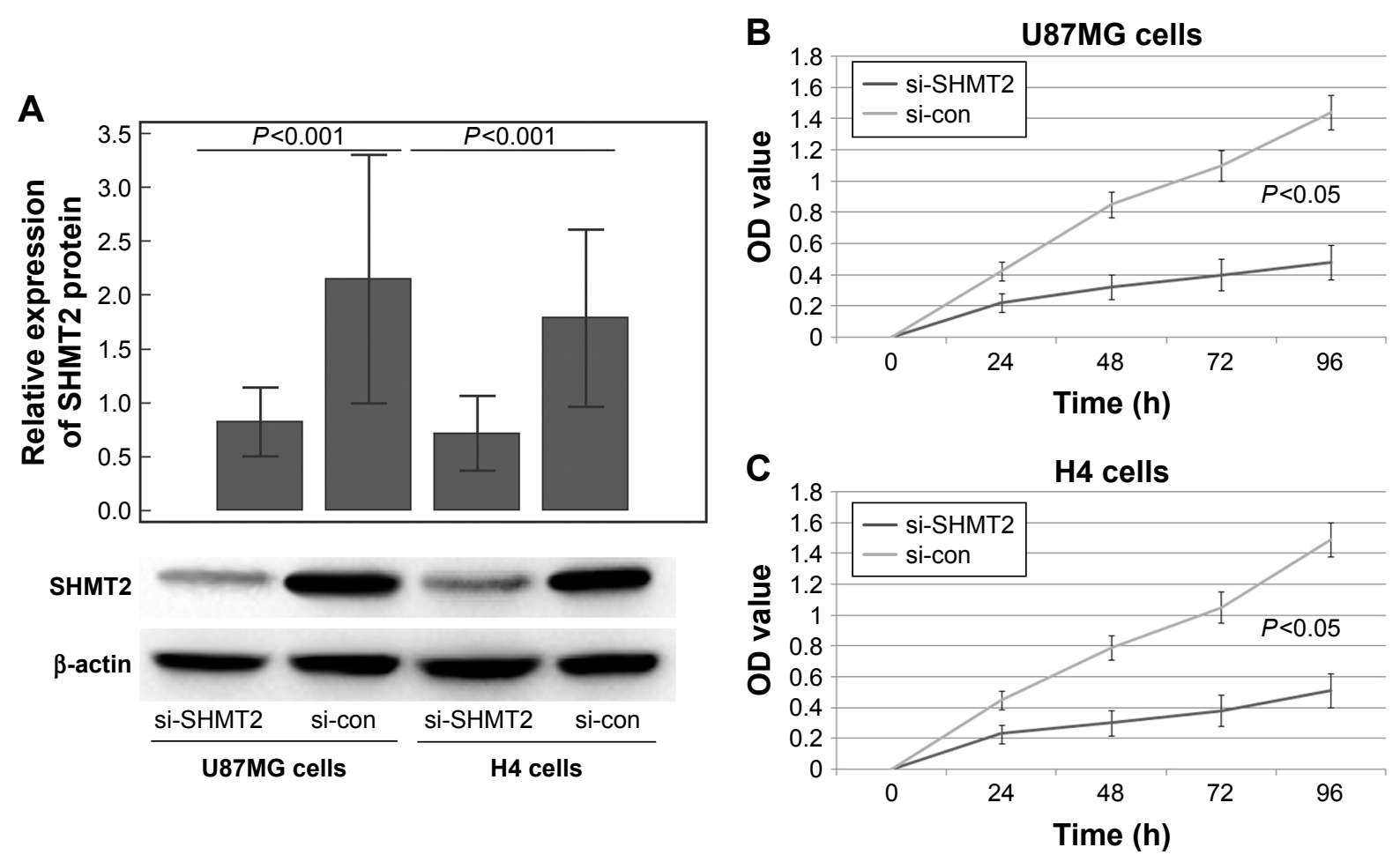

Figure 3 SHMT2 regulates cell proliferation of glioma cells in vitro.

Notes: (A) Expression levels of SHMT2 protein in both si-SHMT2-transfected U87MG and H4 cells were significantly lower than those in si-con-transfected cells based on Western blot analysis. (B and C) After being cultured for $72 \mathrm{~h}$, the cell proliferative activities of si-SHMT2-transfected U87MG and H4 cells were both markedly decreased compared to those of si-con-transfected cells (both $P<0.05$ ).

Abbreviations: SHMT2, serine hydroxyl-methyltransferase 2; si-SHMT2, siRNA to SHMT2; si-con, control siRNA; OD, optical density.

A

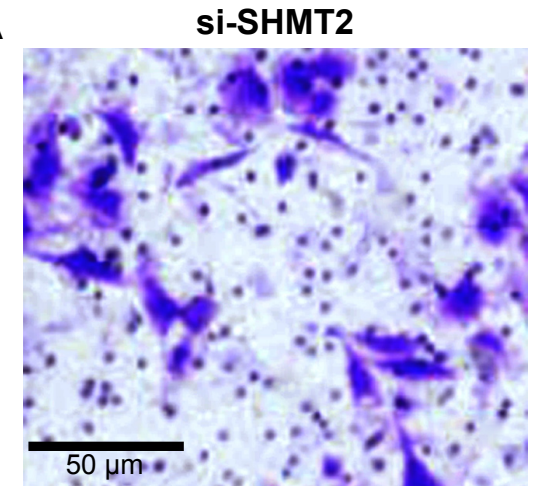

B

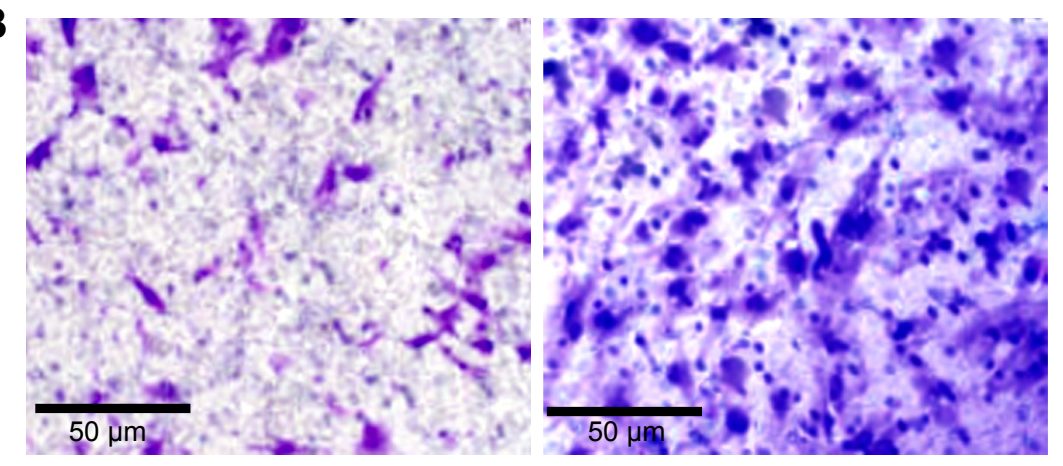

si-con
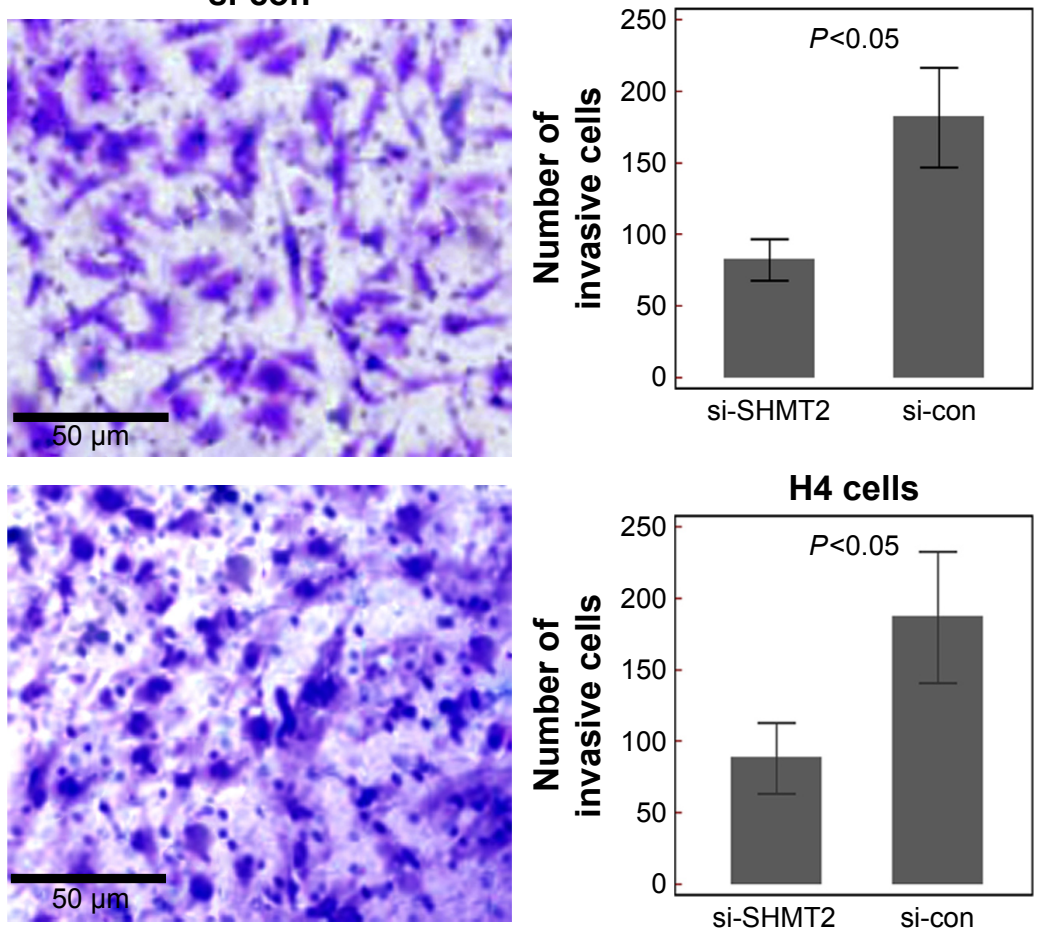

Figure 4 SHMT2 regulates cell invasion of glioma cells in vitro.

Note: (A and B) Loss of SHMT2 expression efficiently suppressed the invasion activities of both U87MG and H4 cells compared with those to those of si-con-transfected cells (magnification 200x).

Abbreviations: SHMT2, serine hydroxyl-methyltransferase 2; si-con, control siRNA; si-SHMT2, siRNA to SHMT2. 
with advanced grade of malignancy $(P<0.001)$ and poor prognosis $(P=0.001)$. Notably, multivariate analysis based on a Cox regression model identified SHMT2 expression as an independent prognostic factor for glioma patients $(P=0.01)$. Functionally, SHMT2 knockdown efficiently suppressed cell proliferation $(P=0.02)$ and cell invasion $(P<0.001)$ of glioma cells in vitro. To the best of our knowledge, this is the first study to confirm the upregulation of SHMT2 with glioma aggressive progression via immunohistochemistry based on a large cohort of clinical samples, combined with the siRNA-induced knockdown assay in vitro.

During the neurological development, the folate cycle is involved in the associations between folate intake and risk for neural tube defects. ${ }^{21}$ Together with serine hydroxylmethyltransferase 1 (SHMT1), SHMT2 functions as a crucial component for the serine/glycine metabolism and transforms serine into glycine in various cells. ${ }^{22}$ Growing evidence shows that the aberrant expression of SHMT2 may be associated with development and progression of several cancer types. Some examples are listed as follows. Zhang et $\mathrm{al}^{8}$ confirmed that SHMT2 overexpression was associated with breast cancer tumor aggressiveness (tumor, node and metastasis [TNM] staging and Elson grade) in a dosedependent manner and the prognostic performance of SHMT2 mRNA was comparable to other gene signatures and proved superior to TNM staging. Woo et $\mathrm{al}^{9}$ reported that SHMT2 inhibition markedly suppressed liver tumorigenesis. $\mathrm{Wu}$ et $\mathrm{al}^{10}$ identified SHMT2 as a direct target gene of miR-615-5p and indicated that knockdown or overexpression of SHMT2 might suppress or promote both proliferation and migration of hepatocellular carcinoma cells; they also determined that SHMT2 expression was dramatically associated with poor prognosis and clinical stage of patients with this malignancy. Lee et $\mathrm{al}^{11}$ performed the comparative oncogenomics analysis and found that elevated expression of SHMT2 might be associated with poor prognosis in human cancer, and functional assays identified SHMT2, a key enzyme in the serine/glycine synthesis pathway, as necessary for tumor cell survival but insufficient for transformation. Wang et $\mathrm{al}^{23}$ observed the overexpression of SHMT2 in glioma, confirmed its promotive role in tumor proliferation and identified this protein as a valuable biomarker for the diagnosis and prognosis of glioma. Consistently, our data revealed the high expression and cytoplasm subcellular localization of SHMT2 in glioma cells of clinical samples, which were demonstrated to be positively correlated with tumor progression and patient's prognosis. Finally, with CCK-8 and transwell assays, we found that the depletion of SHMT2 inhibited proliferation and invasion of both the glioma cell lines (U87MG and H4). However, the previous study of Kim et al ${ }^{12}$ indicated that the knockdown of SHMT2 did not affect the proliferation or survival of U87 cells. The differences between the previous and the current studies may be caused by the heterogeneity of cells in different laboratories. Further validation should be required.

\section{Conclusion}

Our findings suggest that SHMT2 may function as an oncogene in glioma development and progression. Clinically, SHMT2 may serve as a prognostic factor and as a potential therapeutic target for human gliomas. Nevertheless, further research is required to discover the molecular mechanisms of SHMT2 functions in gliomas to pave the way for new therapeutic strategies.

\section{Author contributions}

YX participated in study design and coordination, material support for obtained funding and supervised study. MW performed the experiments and data analysis and drafted the manuscript. SW, XL and QL carried out a part of data analysis. All authors contributed toward data analysis, drafting and critically revising the paper and agree to be accountable for all aspects of the work. All authors read and approved the final manuscript.

\section{Disclosure}

The authors report no conflicts of interest in this work.

\section{References}

1. Ohgaki H. Epidemiology of brain tumors. Methods Mol Biol. 2009;472: 323-342.

2. Bielle F. Building diagnoses with four layers: WHO 2016 classification of CNS tumors. Rev Neurol (Paris). 2016;172(4-5):253-255.

3. Louis DN, Perry A, Reifenberger G, et al. The 2016 World Health Organization Classification of tumors of the central nervous system: a summary. Acta Neuropathol. 2016;131(6):803-820.

4. Li Q, Tu Y. Genetic characteristics of glioblastoma: clinical implications of heterogeneity. Cancer Transl Med. 2015;1(5):176-180.

5. Delgado-López PD, Corrales-García EM. Survival in glioblastoma: a review on the impact of treatment modalities. Clin Transl Oncol. 2016; 18(11):1062-1071.

6. Hebbring SJ, Chai Y, Ji Y, et al. Serine hydroxymethyltransferase 1 and 2 : gene sequence variation and functional genomic characterization J Neurochem. 2012;120(6):881-890.

7. Anderson DD, Stover PJ. SHMT1 and SHMT2 are functionally redundant in nuclear de novo thymidylate biosynthesis. PLoS One. 2009; 4(6):e5839.

8. Zhang L, Chen Z, Xue D, et al. Prognostic and therapeutic value of mitochondrial serine hydroxyl-methyltransferase 2 as a breast cancer biomarker. Oncol Rep. 2016;36(5):2489-2500.

9. Woo CC, Chen WC, Teo XQ, Radda GK, Lee PT. Downregulating serine hydroxymethyltransferase 2 (SHMT2) suppresses tumorigenesis in human hepatocellular carcinoma. Oncotarget. 2016;7(33): 53005-53017.

10. Wu X, Deng L, Tang D, et al. miR-615-5p prevents proliferation and migration through negatively regulating serine hydromethyltransferase 2 (SHMT2) in hepatocellular carcinoma. Tumour Biol. 2016; 37(5):6813-6821. 
11. Lee GY, Haverty PM, Li L, et al. Comparative oncogenomics identifies PSMB4 and SHMT2 as potential cancer driver genes. Cancer Res. 2014;74(11):3114-3126.

12. Kim D, Fiske BP, Birsoy K, et al. SHMT2 drives glioma cell survival in ischaemia but imposes a dependence on glycine clearance. Nature. 2015;520(7547):363-367.

13. Li M, Li X, Xu S, et al. Protein phosphatase 4 catalytic subunit is overexpressed in glioma and promotes glioma cell proliferation and invasion. Tumour Biol. 2016;37(9):11893-11901.

14. Sun J, Zhang L, Zhao H, et al. CHD1L regulates cell cycle, apoptosis, and migration in glioma. Cell Mol Neurobiol. 2016;36(4):565-576.

15. Lu W, Wang X, Liu J, et al. Downregulation of ARHGDIA contributes to human glioma progression through activation of Rho GTPase signaling pathway. Tumour Biol. Epub 2016 Oct 10.

16. Shi ZH, Li XG, Jie WD, Zhao HL, Zeng Y, Liu Y. PTPH1 promotes tumor growth and metastasis in human glioma. Eur Rev Med Pharmacol Sci. 2016;20(18):3777-3787.

17. Lin L, Zheng Y, Tu Y, et al. MicroRNA-144 suppresses tumorigenesis and tumor progression of astrocytoma by targeting EZH2. Hum Pathol. 2015;46(7):971-980.
18. Vigneswaran K, Neill S, Hadjipanayis CG. Beyond the World Health Organization grading of infiltrating gliomas: advances in the molecular genetics of glioma classification. Ann Transl Med. 2015;3(7):95.

19. Wang Y, Jiang T. Understanding high grade glioma: molecular mechanism, therapy and comprehensive management. Cancer Lett. 2013; 331:139-146.

20. Taylor LP. Diagnosis, treatment, and prognosis of glioma: five new things. Neurology. 2010;75(18 suppl 1):S28-S32.

21. Meethal SV, Hogan KJ, Mayanil CS, Iskandar BJ. Folate and epigenetic mechanisms in neural tube development and defects. Childs Nerv Syst. 2013;29(9):1427-1433.

22. Nikiforov MA, Chandriani S, O'Connell B, et al. A functional screen for Myc-responsive genes reveals serine hydroxymethyltransferase, a major source of the one-carbon unit for cell metabolism. Mol Cell Biol. 2002;22(16):5793-5800.

23. Wang B, Wang W, Zhu Z, et al. Mitochondrial serine hydroxymethyltransferase 2 is a potential diagnostic and prognostic biomarker for human glioma. Clin Neurol Neurosurg. 2017;154:28-33.
OncoTargets and Therapy

\section{Publish your work in this journal}

OncoTargets and Therapy is an international, peer-reviewed, open access journal focusing on the pathological basis of all cancers, potential targets for therapy and treatment protocols employed to improve the management of cancer patients. The journal also focuses on the impact of management programs and new therapeutic agents and protocols on

\section{Dovepress}

patient perspectives such as quality of life, adherence and satisfaction The manuscript management system is completely online and includes a very quick and fair peer-review system, which is all easy to use. Visit http://www.dovepress.com/testimonials.php to read real quotes from published authors. 